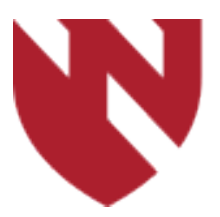

December 2019

\title{
Utilization of Brain Imaging in Evaluating Patients with Psychogenic Nonepileptic Spells
}

\author{
Dangmeng Wei \\ University of Nebraska Medical Center \\ Matthew Garlinghouse \\ University of Nebraska Medical Center \\ Wenyang Li \\ University of Nebraska Medical Center \\ Nicholas Swingle \\ University of Nebraska Medical Center \\ Kaeli K. Samson \\ University of Nebraska Medical Center
}

See next page for additional authors

Tell us how you used this information in this short survey.

Follow this and additional works at: https://digitalcommons.unmc.edu/gmerj

Part of the Higher Education Commons, and the Medicine and Health Sciences Commons

\section{Recommended Citation}

Wei, D., Garlinghouse, M., Li, W., Swingle, N., Samson, K. K., , Taraschenko, O. Utilization of Brain Imaging in Evaluating Patients with Psychogenic Nonepileptic Spells. Graduate Medical Education Research Journal. 2019 Dec 13; 1(1).

https://digitalcommons.unmc.edu/gmerj/vol1/iss1/54

This Conference Proceeding is brought to you for free and open access by DigitalCommons@UNMC. It has been accepted for inclusion in Graduate Medical Education Research Journal by an authorized editor of DigitalCommons@UNMC. For more information, please contact digitalcommons@unmc.edu. 


\section{Utilization of Brain Imaging in Evaluating Patients with Psychogenic Nonepileptic Spells}

\section{Creative Commons License}

\section{(c) (1) $\Theta \Theta$}

This work is licensed under a Creative Commons Attribution-Noncommercial-No Derivative Works 4.0 License.

Authors

Dangmeng Wei, Matthew Garlinghouse, Wenyang Li, Nicholas Swingle, Kaeli K. Samson, and Olga Taraschenko 
an established sensitivity of $80.9 \%$ and specificity of $57.1 \%$ in differentiating high-grade gliomas from tumefactive demyelinating lesions. Some studies suggest that even specimen biopsies may be inconclusive, as there are many histopathologic similarities between tumefactive demyelinating lesions and high-grade gliomas. Diffusion tensor imaging (DTI), though not yet utilized in common practice, can be highly sensitive in differentiating these lesions.
Conclusions: For both patient scenarios, MR imaging was insufficient to make a definitive diagnosis, resulting in a delay in treatment. The cases and literature review demonstrate the diagnostic challenges of these conditions using standard MRI alone, and raise awareness of DTI and other non-invasive modalities that may become integrated into future clinical practice.

https://doi.org/10.32873/unmc.dc.gmerj.1.1.053

\section{Utilization of Brain Imaging in Evaluating Patients with Psychogenic Nonepileptic Spells Danmeng Wei, Matthew Garlinghouse, Wenyang Li, Nicholas Swingle, Kaeli K. Samson, Olga Taraschenko}

Mentor: Olga Taraschenko

Program: Neurology

Background: Psychogenic non-epileptic spells (PNES) are paroxysmal movements or sensory events that resemble epileptic seizures but lack corresponding ictal electrographic changes. Patients are often referred for brain imaging tests which contribute to the high cost of care.

Methodology: This is a retrospective chart review of 225 adult patients diagnosed with PNES without epileptic seizures from 2012 to 2017. The frequency of the brain imaging tests prior to vEEG was assessed across all semiological classes and was correlated with other clinical characteristics.

Result: The most prevalent PNES events were characterized by semi-rhythmic small amplitude movements in the extremities (32\%) followed by those resembling clonictonic seizures $(27.7 \%)$. Patients with sensory changes had more imaging tests than those with primitive gesturing and axial posturing. Patients with 3 or more psychiatric disorders had more combined MRIs and CTs prior to diagnosis than patients with two or fewer psychiatric co-morbidities $(\mathrm{p}=0.03)$.

Conclusion: The frequency of brain imaging obtained prior to the definitive diagnosis of PNES is influenced by semiology and the psychiatric health of patients. The PNES with minimal paroxysmal movements in the settings of multiple psychiatric co-morbidities represent particularly challenging patient phenotype which is linked to excessive referrals for brain imaging.

https://doi.org/10.32873/unmc.dc.gmerj.1.1.054

\section{Disseminated Strongyloides Causing Diffuse Alveolar Hemorrhage Mollie Brittan, Brian Boer, Deepak Chandra}

\section{Mentor: Brian Boer}

Program: Internal Medicine

Case presentation: An elderly patient with a past medical history of leukocytoclasic vasculitis on high dose steroids presented with altered mental status. The patient was found to be in septic shock and was admitted to an intensive care unit. The patient was started on broad spectrum antibiotics but continued to decline and developed hemoptysis along with hypoxic respiratory failure requiring intubation. Bronchoscopy revealed frank bronchial and alveolar hemorrhage. Bronchoscopy fluid was sent for microscopy and it revealed nematodes consistent with Strongyloides. The patient was started on

\section{Was It the Beer or the Burgers?} Ian Cormier, Craig Baumgart, Michael Smith

\section{Mentor: Michael Smith \\ Program: Internal Medicine}

Acute pancreatitis is a commonly encountered diagnosis and is the most common gastrointestinal reason for hospitalization. Approximately $75 \%$ of cases of acute pancreatitis are caused by gallstones oral Ivermectin and Albendazole but failed to improve on this regimen and was switched to subcutaneous Ivermectin after FDA approval. The patient ultimately improved and was discharged.

Discussion: Strongyloides stercolis is a nematode that can cause disease in humans. Strongyloides infection can involve cutaneous, pulmonary and gastrointestinal systems. This organism has a unique lifecycle where the parasite enters the host often through the skin and migrates to both the gastrointestinal and pulmonary systems at various points during its lifecycle. This unique life cycle also involves gastrointestinal autoinfection. Autoinfection occurs when a human is infected with a helminth (i.e.
Strongyloides) and the whole parasite's lifecycle takes place within the human host. This process is exaggerated in humans when cell mediated immunity is impaired with immunosuppression (in the case of our patient with corticosteroids). It has the ability to spread to multiple organs during the hyperinfection stage which can cause multisystem organ failure.

Conclusion: Diffuse alveolar hemorrhage caused by disseminated Strongylioides has a high mortality rate and treatment with subcutaneous Ivermectin contributed to her survival as the oral formulation had poor absorption due to gastrointestinal involvement of the parasite.

https://doi.org/10.32873/unmc.dc.gmerj.1.1.055 or alcohol consumption. In contrast, hypertriglyceridemia only accounts for $2-4 \%$ of acute pancreatitis cases.

A middle-aged patient with a history of recurrent acute pancreatitis secondary to alcohol abuse was admitted for acute epigastric abdominal pain consistent with his prior episodes of pancreatitis. Workup was significant for multiple electrolyte derangements and laboratory workers commented on the patient's serum appearing lactescent. Repeat point-of-care testing revealed normal electrolyte levels. A lipid panel revealed profound hypertriglyceridemia and the patient was treated with an insulin 